\title{
Identification of modes in main-sequence pulsators by means of multi-color photometry
}

\author{
J. Daszyńska-Daszkiewicz ${ }^{1,2}$, W.A. Dziembowski ${ }^{3,4}$, A.A. Pamyatnykh ${ }^{4,5,6}$ \\ ${ }^{1}$ Instituut voor Sterrenkunde, Celestijnenlaan 200B, 3001 Leuven, Belgium \\ 2 Astronomical Institute of the Wrocław University, ul. Kopernika 11, 51-622 Wrocław, Poland \\ 3 Warsaw University Observatory, Al. Ujazdowskie 4, 00-478 Warsaw, Poland \\ 4 Copernicus Astronomical Center, ul. Bartycka 18, 00-716 Warsaw, Poland \\ 5 Institute of Astronomy, Russian Academy of Science, Pyatnitskaya Str. 48, 109017 Moscow, Russia \\ ${ }^{6}$ Institute of Astronomy, University of Vienna, Türkenschanzstr. 17, A-1180, Vienna, Austria
}

\begin{abstract}
We discuss two problems connected with the application of the method of photometric amplitudes and phases for mode identification. The first one concerns the effect of rotational mode coupling in $\beta$ Cep models. The second one deals with the strong dependence of the photometric observables on convection in $\delta$ Scuti stars.
\end{abstract}

\section{Introduction}

Oscillation spectra of main-sequence pulsators, like $\beta$ Cep and $\delta$ Sct stars, only seldom exhibit equidistant patters, which are typical, for example, in white dwarfs. Therefore, it is difficult to identify oscillation modes from frequency spectra alone. The most popular method of mode identification is based on multicolor photometry (Balona \& Stobie 1979, Watson 1988). We address here two problems concerning this method which have been discussed in details in two of our recent papers (Daszyńska-Daszkiewicz et al. 2002, 2003).

\section{Photometric amplitudes and phases in rotating $\beta$ Cep models}

If effects of rotation are negligible, the photometric diagnostic diagrams (amplitude ratio vs. phase difference) are independent of the inclination angle, $i$, and of the azimuthal order, $m$, and they may reveal the spherical harmonic degree, $\ell$. For $\beta$ Cep star models, oscillation modes are located in the diagnostic diagrams in very well separated regions of different values of $\ell$. With the results of linear nonadiabatic theory these diagrams have been successfully applied to many stars (Cugier et al. 1994).

However amongst $\beta$ Cep stars we meet quite often fast rotators. The most important effect of moderate rotation is mode coupling. It occurs if the frequency difference between modes $j$ and $k$ is of the order of the angular velocity of rotation, $\omega_{j}-\omega_{k} \sim \Omega$, and if the spherical harmonic indices satisfy the relations: $\ell_{j}=\ell_{k} \pm 2$ and $m_{j}=m_{k}$. For eigenfunctions of coupled modes one has to consider superpositions of all modes satisfying the conditions mentioned above. The complex amplitude of the monochromatic flux variation for a coupled mode is expressed as

$$
\mathcal{A}_{\lambda}(i)=\sum_{k} a_{k} A_{\lambda, k}(i)
$$



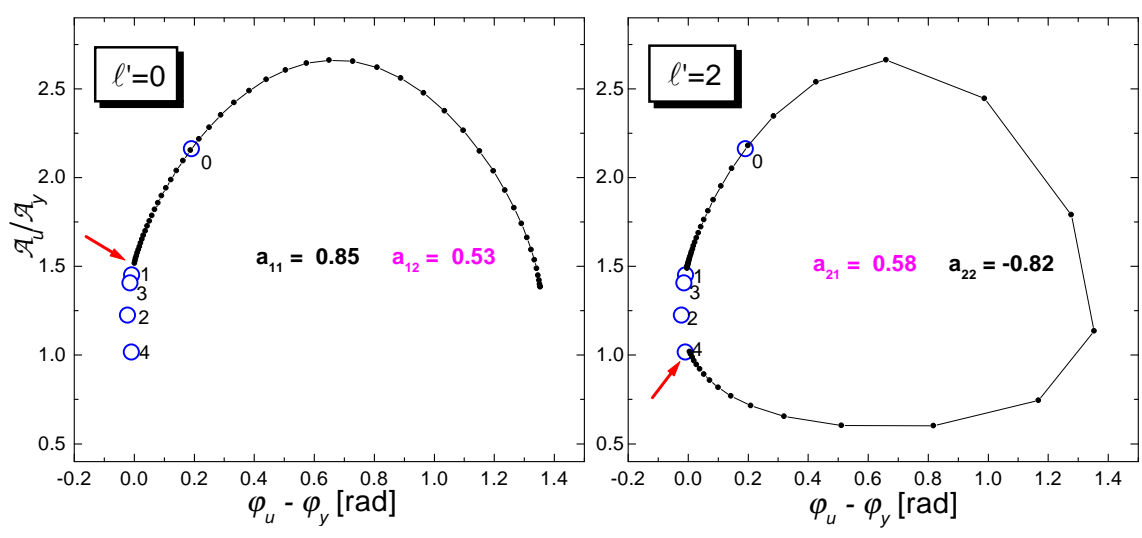

Figure 1: Two mode coupling between $\ell=0, p_{1}$ and $\ell=2, g_{1}$ with the dimensionless frequency distant $\Delta \sigma=0.003$, in the model at $\log T_{\text {eff }}=4.374$ and $V_{\text {rot }}=100 \mathrm{~km} / \mathrm{s}$. Arrows point the polar direction.

where $a_{k}$ are solutions of the degenerate perturbation theory for slowly rotating stars (Soufi et al. 1998). These quantities describe contributions of $k$ th- spherical harmonic to the coupled mode. $A_{\lambda, k}$ is the complex amplitude for single- $\ell$ mode. Now the amplitude ratios and phase differences become both $i$ and $m$ dependent.

In Fig. 1 we give an example of coupling between a close $\ell=0$ and 2 pair at an equatorial velocity of about $100 \mathrm{~km} / \mathrm{s}$. Open circles mark positions of single $\ell$ modes in the diagram based on $u$ and $y$ Strömgren passbands. In the left panel, we see the nominal $\ell=0$ mode. It is indeed dominated by the $\ell=0$ component, but there is a considerable $\ell=2$ component. Small dots are equally spaced in $\cos i$, therefore their density reflects the probability of the situation. In the right panel we see the behavior of the nominal $\ell=2$ mode. As one can see, both modes can lie in various parts of the diagnostic diagrams. It may happen that both modes can be identified as an $\ell=1$ or $\ell=0$ doublet. We can find also positions where single- $\ell$ modes never appear.

\section{Constraints on convection from multi-color photometry of $\delta$ Sct stars}

In $\delta$ Scuti star models, the photometric amplitudes and phases exhibit a strong dependence on convection. Calculations of these observables make use of the complex parameter $f$, which gives the ratio of the radiative flux perturbation to the radial displacement in the photosphere. This parameter is obtained with the linear nonadiabatic theory, and is very sensitive to convection, whose treatment still remains rather uncertain.

The strong sensitivity of calculated mode positions in the diagnostic diagrams to treatment of convection is not necessarily bad news. Having data from more than two passbands we may try to determine simultaneously $\ell$ and $f$. If we succeed, the $f$-value inferred from the data can yield a valuable constraint on models of stellar convection.

We (Daszyńska-Daszkiewicz et al. 2003) proposed the method based on $\chi^{2}$ minimization assuming trial values of $\ell$. We regard the $\ell$ degree and the associated complex $f$ value as the solution if it corresponds to $\chi^{2}$ minimum which is much deeper than at other values of $\ell$. As the next step, we generalized this method to the case of modes coupled by rotation, and we extended it by adding the radial velocity measurements. Additionally, in the case of a radial mode our method allows to refine the stellar global parameters, by fitting them to reproduce the exact value of the observed frequency. Up to now we applied this method to four stars: 

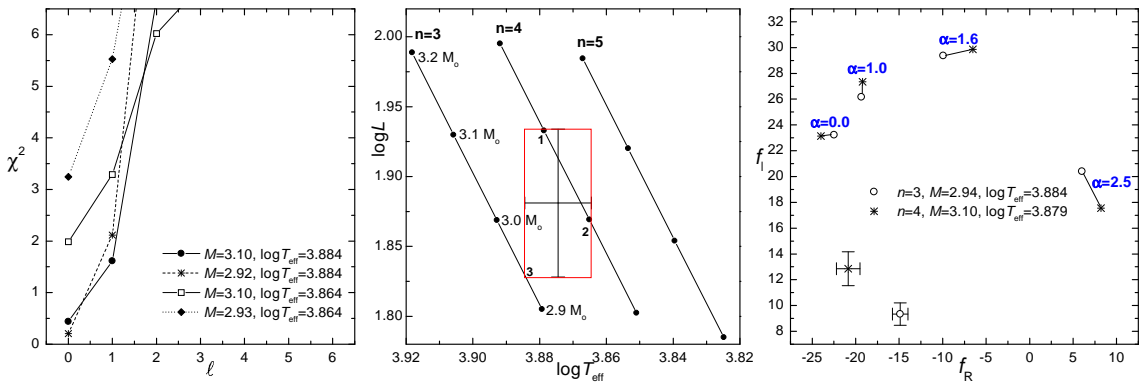

Figure 2: Left panel: $\chi^{2}$ as a function of $\ell$ for four models of $20 \mathrm{CVn}$. Middle panel: The HR diagram with the observational error box for $20 \mathrm{CVn}$ and with lines of the constant periods for $n=3,4,5$. Only models along these lines are allowed. Right panel: comparison of empirical values of the $f$ parameter with theoretical ones calculated for four values of the MLT parameter, $\alpha$.

1. $\beta$ Cas, an example with a possibly coupled mode,

2. $20 \mathrm{CVn}$, an example with tuning of stellar parameters (see example in Fig. 2),

3. AB Cas, an example with large uncertainties in stellar parameters,

4. 1 Mon, an example of combining photometry and spectroscopy.

\section{Conclusion}

Fast rotators occur quite often among $\beta$ Cephei stars. Rotation complicates usage of photometric diagnostic diagrams for mode identification. It causes that there are more candidates for mode identification from multi-color photometry, because modes with higher $\ell$ may acquire large low degree components. Moreover coupled modes are aspect- and $m$-dependent. Therefore the mode identification in fast rotating stars may be done only simultaneously with determination of stellar parameters and the inclination of rotational axis.

Application of multi-color photometry of $\delta$ Scuti stars goes beyond the identification of the spherical harmonic degree, $\ell$. We proposed a new method of extracting the nonadiabatic parameter, $f$, from such data (Daszyńska-Daszkiewicz et al. 2003). The method yields constraints on stellar convection. Determination of $\ell$ is an independent goal. It can be done without a priori knowledge of $f$. Moreover, if the observed mode is radial we are able to diminish significantly the uncertainty of stellar parameters. Finally, we proposed for the first time a way how to combine photometry and spectroscopy for mode identification, adding radial velocity data to our method.

Acknowledgments. The work was supported by the Polish KBN grant No. 5 P03D 012 20. J. D-D. gratefully thanks the Instituut voor Sterrenkunde in Leuven for financial support for the JENAM 2003 conference.

\section{References}

Balona L. A., Stobie R.S., 1979, MNRAS, 189, 649

Cugier H., Dziembowski W. A., Pamyatnykh A. A., 1994, A\&A, 291, 143

Daszyńska-Daszkiewicz J., Dziembowski W. A., Pamyatnykh A. A., Goupil M-J., 2002, A\&A 392, 151

Daszyńska-Daszkiewicz J., Dziembowski W. A., Pamyatnykh A. A., 2003, A\&A 407, 999

Soufi F., Goupil M-J., Dziembowski W. A., 1998, A\&A 334, 911

Watson R. D. 1988, Ap\&SS 140, 255 